\title{
Sophisticated Microwave Nondestructive Testing Method for Metals
}

\author{
Vladimir Sutorikhin ${ }^{1}$ \\ ${ }^{1}$ Remote Indicators of Active Defects (RIAD) Ltd. Co., 12/2 - 46 Nakhimov Road, Tomsk, Russia \\ Correspondence: Vladimir Sutorikhin, Remote Indicators of Active Defects (RIAD) Ltd. Co., 12/2 - 46 Nakhimov \\ Road, P.O. Box 634012 Tomsk, Russia. Tel: 8-382-254-1261, 8-913-824-4039. E-mail: wind@mail.tomline.ru
}

Received: June 21, 2012 Accepted: August 9, 2012 Online Published: October 10, 2012

doi:10.5539/apr.v4n4p8 URL: http://dx.doi.org/10.5539/apr.v4n4p8

\begin{abstract}
The present paper describes theoretically and experimentally the possibility of such electron gas interaction with low-frequency ultrasonic waves, which the authors determine as a newly revealed phenomenon or even discovery, changing the physical concept of metal destruction processes and metal ageing. In contrast to previous experiments in determining surface cracks by MW method, the author has produced new experimental data in identifying invisible metal defects, i.e. active defects. Although the described method is based on the well-known Acoustic Emission (AE) method, there are significant differences. Firstly, according to calculations and experiment results the propagation speed of active defects generated by this new method is close to the speed of light. Secondly, in contrast to single AE events, occurring under conditions of excessive mechanical force, displayed signals in this new method can be detected during a time-interval excluding an additional mechanical load. Finally, the non-contact detection of dangerous defects, i.e. active defects, indicates the advantageous aspect of this new method to the contact AE method. This paper presents experimental data, sustaining the possible practical application of an advanced remote indicator of active defects (RIAD) as a diagnostic technique for steam railway car wheels in motion mode, testing reliability parameters of rotating turbine blades and operating sucker rod pumps.
\end{abstract}

Keywords: active defects, ultrasonic waves, MW method, remote indicator of active defects (RIAD), Acoustic Emission (AE) method

\section{Introduction}

One of the modern NDT techniques for inspection and monitoring metal objects is Acoustic Emission (AE), the fundamental physical principles of which describe the initiation and extension of micro-cracks as right in the moment occurrence and merging of numerous dislocations within a polycrystalline body (Dudova, Kaibishev, Valitov, 2008). Such sources as mechanical loading and thermal stresses generate high mobility and further multi-dislocations, which in its turn, form into micro-cracks. These processes are visually observed during loading (Shibkov \& Zolotov, 2011). In the following case, the investigation of dislocation mobility processes includes such instrumentation as contact piezoelectric converters, as well as, optical methods. A notable breakthrough in this area could be considered the research conducted by Tomsk scientists in applying MW frequencies of 9.8 and $28 \mathrm{MHz}$ to monitor definite AE parameters (Gorbunov, Sutorikhin, \& Ustinov, 2008).

Different variants of laboratory equipment were designed and tested during the research period. In spite of the visible formation process of elastic compression-rarefaction waves, either in extension or on metal surface, the possible registration of electrical conductance variable components directly coupled density was excluded during propagation of acoustic signals. Nevertheless, during the past few decades studies about determining defects (cracks) on metal surface by microwaves (MW) have been performed. A Japanese team worked with a coaxial probe operating at high frequencies to detect surface cracks of resolution 1-3 microcrones $(\mu \mathrm{m})$. The resonator included an open coaxial line (waveguide) with Transverse Electric and Magnetic (TEM) mode (i.e. micro-coaxial cables with $1 \mathrm{~mm}$ cross-section). This technique allows significant increase of the resolution at $110 \mathrm{GHz}$ and decrease of the coaxial cable cross-section to improve operating parameters obtained in the experiment. This experimental device detects surface cracks of $0.125 \mathrm{~mm}$ at a distance of $0.5 \mathrm{~mm}$ (Ju, Saka, \& Luo, 2002).

In this paper an entirely new unknown alternative MW method is proposed in the identification of initial destruction and registration of active defects in and on the surface of a metal. In contrast to surface phenomena 
studies, such as surface cracks, Chernov-Luders bands, the proposed method involves an advanced physical phenomenon-interaction of electron gas and ultrasonic waves in metals (frequency density up to $5 \mathrm{~W} / \mathrm{cm}^{2}$ ), at any point on the metal surface where defects are formed, reflecting the energy i.e. generating ultrasonic impulses.

The first experiments were conducted on ST 4 brand-steel and ST 45 brand-steel samples, i.e. "electro-technical transformer steel", in tension testing device, which led to further testing experiments with other samples aluminum and graphite. All obtained experimental results were executed as reports or scientific papers (Gorbunov \& Sutorikhin, 1999, 2010, 2012).

The present paper describes theoretically and experimentally the possibility of such electron gas interaction with low-frequency ultrasonic waves, which the authors determine as a newly revealed phenomenon or even discovery, changing the physical concept of metal destruction processes and metal ageing. This paper presents experimental data, sustaining the possible practical application of an advanced remote indicator of active defects (RIAD) for metallic units and construction designs.

\section{Description of Detected Phenomenon}

During a routine testing in determining acoustic emission parameters, MW sensor response to generated ultrasonic signal was revealed. The following experiment was conducted in tension testing device with maximum loading 10 tons (frequency $100 \mathrm{KHz})$. The testing tension sample - steel metal plate surface $(3 \mathrm{x} 45 \mathrm{x}$ $450 \mathrm{~mm}$ ) involves ultrasonic wave frequency $54 \mathrm{KHz}$, while output is not more than $5 \mathrm{~W} / \mathrm{cm}^{2}$. Microwave (MW) sensor with frequency $28 \mathrm{GHz}$ (designed in accordance to Doppler radar) was perpendicularly focused on the investigated sample plate surface at a distance of $100-120 \mathrm{~mm}$. Furthermore, spectrum analyzer system SK4-59, plugged into the microwave (MW) sensor outlet, could not register any displayed signal level (ultrasonic frequency $54 \mathrm{KHz}$ ) of sample surface before the operating performance of tension testing device. However, it was established that under imposed load close to elastic tension, a sudden increase of displayed signal level was registered of 26-30 decibels above noise level.

The major oscillation type is flexural waves under the following conditions-ultrasonic frequency of $54 \mathrm{KHz}$ and plate thickness of $3 \mathrm{~mm}$. Measured stationary wave length of such oscillations is $120 \mathrm{~mm}$, corresponding to $\mathrm{S}$-wave propagation speed of $4.84 \mathrm{~km} / \mathrm{sec}$. Measured stationary wave (S-wave, flexural wave) amplitude is determined by piezoconverter (MA40E9-1), where response is minus 86 decibel (relative 0 decibel $=10 \mathrm{~V} / \mathrm{Pa}$ ) and includes $200 \mathrm{mV}$. As a result, the pressure, developing on metal surface to ultrasonic exposure, is 50 times less than $1 \mathrm{~Pa}$, i.e. $0.02 \mathrm{~Pa}$. The amplitude of $0.02 / 3 \mathrm{E} 6=6.5 \mathrm{E}-9(\mathrm{~m})=6.5$ nanometer is obtained at pressure $0.02 \mathrm{~Pa}$ and $3 \mathrm{MPa} / \mathrm{m}$ in Young modulus (for ceramics). At oscillation amplitude of 6.5 nanometers and microwave field frequency of $28 \mathrm{GHz}$ (wave length $10.7 \mathrm{~mm})$ relative MW phase change $(20 \log ((65 E-7) / 10,7)=-124,6$ decibel) waves are not more than minus 124.6 decibel. Taking into account the fact that phase differential $\pi / 2$, calculated relative phase differential value for oscillation amplitude surface of 6.5 nanometers, the maximum MW phase change signal is

$$
\text { 124.6-12.04 (4 times) }=112.56 \text { decibel. }
$$

Considering the influence of prototype MW sensor noise coefficient, maximum response value was calculated based on the measured values of generator noise phase - 13 decibel and generator noise amplitude- 12 decibel to Gunn diode as [(13 decibel ) 20 (times) $+(12$ decibel $) 15.8$ (times) $=(15.5$ decibel $) 35.8$ (times)]. Having calculated the maximum constant component value to operating load of MW frequency, diode (3000 Ohm) $\left(R_{H}\right)$ 200-250 $\mathrm{mV}((U)$, initial approximation could be maximum signal energy)

$$
P=U^{2} / R_{u}=20 \mathrm{E}-6 \mathrm{~W},=-16.8 \mathrm{decibel} / \mathrm{mW}
$$

corresponds to the energy in phase difference $\pi / 2$. In this case, it is impossible to observe the modulated signal energy

$$
-16.8-112.56=-129.36 \mathrm{decibel} / \mathrm{mW}
$$

on the spectrum analyzer SK4-59, where registration frequency band- $1000 \mathrm{~Hz}$, temperature-300K and noise coefficient 10 decibel. In fact, noise adjusted at spectrum analyzer inlet is equal to the noise at MW sensor outlet (with 13 decibel amplification) plus analyzer noise at the inlet itself. As a result, the basic noise (MW converter noise coefficient-12 decibel), Gunn generator noise (15.5 decibel), including converter amplification factor (13 decibel), analyzer noise at inlet ( 10 decibel) are equal $(35.8+15.8) \times 20+10=1042(30.2$ decibel). Increasing noise energy relative to maximum value, defined cascade Friis formula (Vondran, 1999) (144 decibel/mW to KHz, at $300 \mathrm{~K})$ is

$$
-144+30.2=-113.8 \text { decibel } / \mathrm{mW}
$$


Thus, it is impossible to observe the signal level of 129.36 decibel $/ \mathrm{mW}$ (3). The signal proves to be lower than the noise level (113.8-129.35) $=-15.55$ decibel (36 times). Calculation error (noise coefficient calculation) is not more than 3 decibel.

In the experiment, the observed displayed signal level exceeded the noise up to 30 decibel. In this case, there are two assumptions: (1) surface oscillation amplitude exceeds the measured value by 45.55 decibel $[15.55+30=$ 45.55 decibel, (190 times)]

$$
\text { and is as follows [(6.5 E-6) }(190)=1235 \mathrm{E}-6 \mathrm{~mm},(1.24 \text { micron })]
$$

(2) phase differential is the result of alternating surface conduction, occurring at ultrasonic frequency of $54 \mathrm{kHz}$, practically simultaneously all over this metal surface.

The first assumption should be rejected as the ultrasonic energy output in the experiment was not more than $5 \mathrm{~W} / \mathrm{cm}^{2}$ and could not abruptly change to 45.55 decibel (190 times). The second assumption does not contradict energy conservation law. Potential MW signal modulation due to surface conductance alteration with ultrasonic frequency is undisputable. However, it is inevitably assumed that alternating surface conduction is related to density or temperature changes, provided that wave density should correspond to ultrasonic wave length. It was established that the wave length variable surface conduction is significantly higher than that of the ultrasonic one (>1000 times). The experiments in determining the functional dependency value between displayed signal and exposed surface location showed that any investigated sample regionally reflects MW to the sensor, leading to displayed signal. Ultrasonic component spectrum amplitude does not actually depend on the sensor location.

Even today this problem has not been solved, notwithstanding the numerous conflicting explanations. It is the variety of explanations that makes it impossible to choose the accurate concept in this case. Nevertheless, the newly revealed phenomenon, as result of a 15 -year experimental research, could be the only answer to this question. Our experiments not only gave adequate support for previous theoretical conclusions, but also proved the practical application of this phenomenon, i.e. sophisticated prototype device-remote indicator of active defects on metal surface, determining units durability, either in motion or under dielectric layer.

\section{Theoretical Background}

It is presupposed that the significant MW field modulation level increase is interconnected with the anomaly metal "skin layer", changing its properties under defects and associated with the beginning of micro-crack formation. It is established that the electric $\boldsymbol{E}_{t}(0)$ and magnetic $\boldsymbol{H}_{t}(0)$ field ratio on metal surface is written as Leontovich impedance approximation (Leontovich M.A., 1948).

$$
\boldsymbol{E}_{t}(0)=\sec . / 4 \pi Z(\omega, \sigma)\left[\boldsymbol{H}_{t}(0) \times \boldsymbol{n}\right]
$$

where: sec.- speed of light $\left(3 \times 10^{8} \mathrm{~m} / \mathrm{sec}\right.$.); $Z(\omega, \sigma)$-surface (skin) impedance (sec./m); angular frequency and conduction functions; n-normal vector to surface; $\omega$-angular frequency (rad./sec.) and $\sigma$ - metal electrical conduction $\left(10^{10}-10^{17} 1 / \mathrm{sec}\right.$.) - amplitude of surface (skin) impedance.

Comparing above-mentioned equation (6) to Maxwell equation (Bredow, Rumyantsev, \& Toptigin, 1985) it can be written as:

$$
Z(\omega, \sigma)=4 \pi / c \varepsilon^{1 / 2}(\omega, \sigma), \varepsilon(\omega, \sigma)=\varepsilon_{l}+j 4 \pi \sigma / \omega
$$

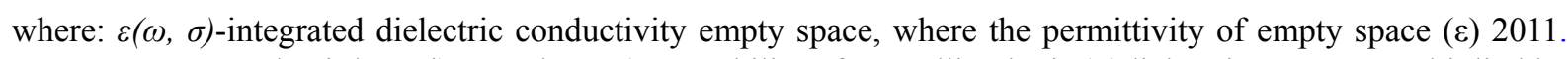
www.answers.com/topic/permi); $\varepsilon_{l}$ real part (permeability of crystalline lattice)( dielectric constant multiplied by the permittivity of

Surface impedance may be written as reflection coefficient $\rho$ :

$$
\rho=\boldsymbol{H}_{t}(\mathrm{x}) / \boldsymbol{H}_{t}(0)=(1-c Z / 4 \pi) /(1+c Z / 4 \pi)
$$

By substituting (7) into (8), we obtain:

$$
\rho=\left(\varepsilon^{1 / 2}(\omega, \sigma)-1\right) /\left(\varepsilon^{1 / 2}(\omega, \sigma)+1\right)
$$

Given formulae, describing "normal skin effect", are valid in terms of surface reflection homogeneity. This homogeneity factor is unequal on "skin layer" thickness $\left(\delta_{0}\right)$

$$
k<\delta_{0}=\text { sec. } /\left(2 \pi(f \sigma)^{1 / 2}\right) .
$$

It follows that spatial wave field heterogeneity at a distance of electron free path $(l)$ should be insignificant. If only this factor is not considered, then spatial wave field heterogeneity becomes significant (during time 
collision the electron "speeds" through that zone where field microwaves have different values). In most cases, defects are determined under conditions of normal or increased temperatures (300-900 K). Inequality (10) at these given temperatures is satisfied to infrared frequency band.

Determining MW field reflection coefficient by integrated dielectric conduction (7) shows that value for constant frequency $(\omega=$ const) remains conduction $\sigma$. Substituting $\varepsilon(\omega, \sigma)$ from (7) into (10), involving the square root and combining real and imaginary parts, we obtain:

$$
\sqrt{\varepsilon(\omega, \sigma)}=+\sqrt{\bmod (\varepsilon(\omega, \sigma))} \exp (\varphi(\varepsilon(\omega, \sigma)) / 2,
$$

negative square root is excluded,

$$
\begin{gathered}
\bmod (\varepsilon(\omega, \sigma))=\sqrt{\varepsilon_{l}^{2}+(2 \sigma / f)^{2}}=A, f=\omega / 2 \pi, \varphi(\varepsilon(\omega, \sigma))=\arctan \left(2 \sigma /\left(\varepsilon_{l} f\right)\right)=2 B, \\
\rho=((\cos (B)-1 / \sqrt{A})+j \sin (B)) /((\cos (B)+1 / \sqrt{A})+j \sin (B)) \text { at } \sqrt{A} \triangleright 0 .
\end{gathered}
$$

The imaginary part ratio $\phi(\rho)$, determining the wave reflection phase, is of special interest:

$$
\varphi(\rho)=\arctan (\sin (B) /(\cos (B)-1 / \sqrt{A}))-\arctan (\sin (B) /(\cos (B)+1 / \sqrt{A}))
$$

Substituting numerical values $\sigma / f=(10), \varepsilon_{l}=100$ is equally valid and acceptable as $\varepsilon(\omega, \sigma)=\infty$, in spite of the established value $\varepsilon(\omega, \sigma)=1$ for metals. This was described in details by B.B. Golitsin (Golitsin B.B., 1960), based on Cohn conclusions. According to the calculations of Zaev, Avramenko and Lisin (2012), real part of metal dielectric component is up to $10^{8}$. Above-mentioned values are used in the calculations, where ratio percentage $2 \sigma / f$ changes. In this case we obtained the following: value graph of the reflection coefficient (Figure 1 conduction alteration function from 1 to $16 \%$ ); calculation value of displayed signal above noise (Figure 2 at noise level - 113 decibel, maximum displayed signal level - 17 decibel $/ \mathrm{mW}$ ) and calculation value of desired signal (Figure 3 relative value of reflection coefficient modulus at $2 \sigma / f=10$ ).

Based on these calculations it can be stated that the alteration $\sigma$ is $10 \%$ (for example, from $5 \times 10^{11}$ to $5.5 \times 10^{11}$ $(1 / \mathrm{sec})$.$) and leads to the modulation of the wave reflection up to 24$ decibel (signal/noise) (Figure 2 relative maximum value for $\pi / 2$, which is comparable to experimental results. In practical terms, there is an increase above noise up to 30 decibel, consequently, conduction changes up to $17-18 \%$ (Figure 2).

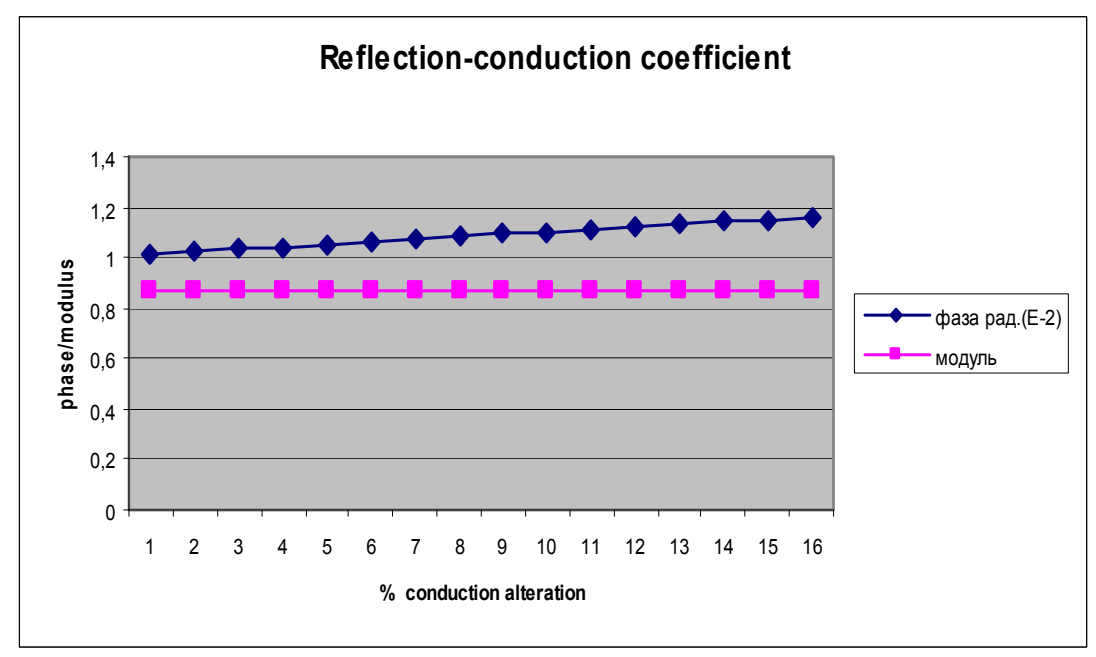

Figure 1. Reflection-conduction coefficient $2 \sigma / f=10, \varepsilon_{l}=100$ 


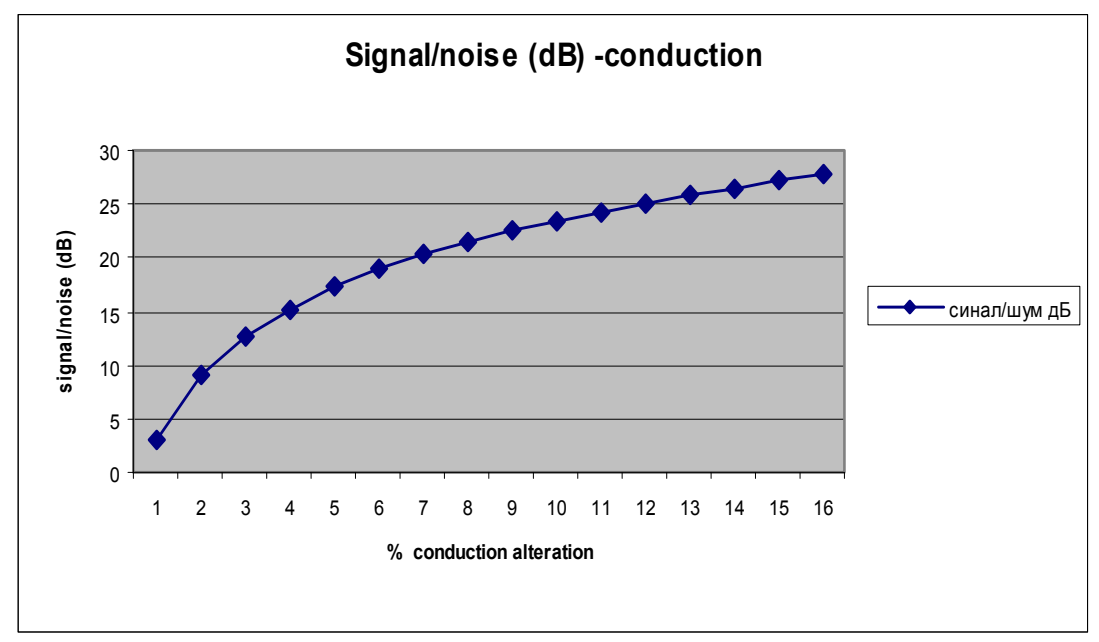

Figure 2. Signal/ noise (decibel)-conduction $2 \sigma / f=10, \varepsilon_{l}=100$, noise level-113 decibel $/ \mathrm{mW}$

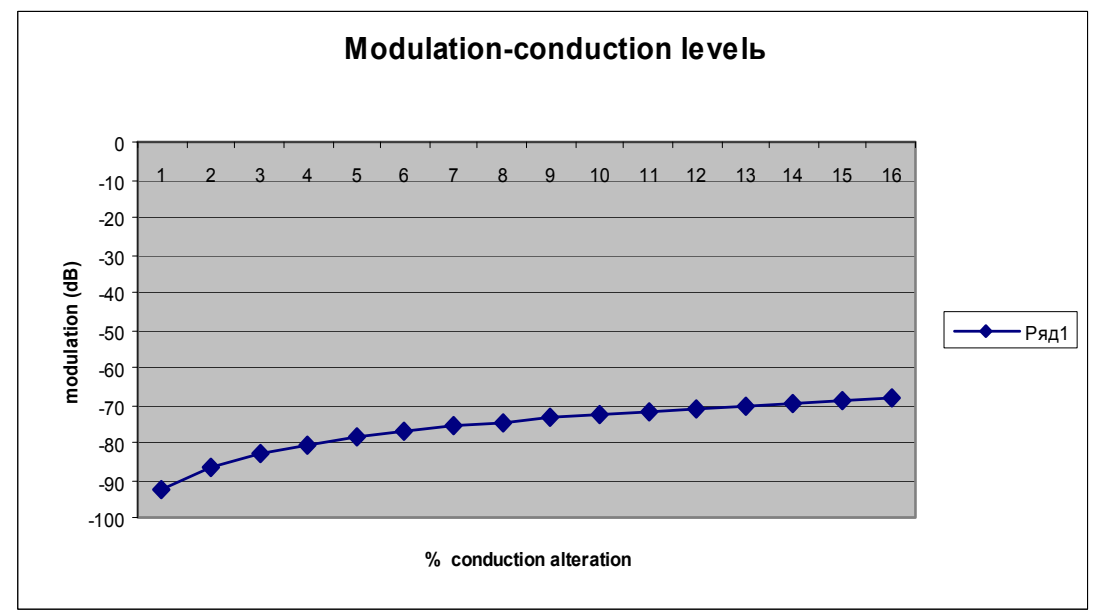

Figure 3. Displayed signal (decibel) $2 \sigma / f=10, \varepsilon_{l}=100$

It should be noted that the measurement of displayed signal level occurs at ultrasonic energy of $5 \mathrm{~W} / \mathrm{cm}^{2}$. Changes of this energy could alter the results of performed calculations.

Performed calculations in accordance to stated formulae showed the fact that the most simple and reliable explanation is increased modulation level on metal surface with defects - "skin layer" conduction level alters to ultrasonic response frequency.

In this case it is rather difficult to explain the surface conduction alteration behavior by low-frequency ultrasonics. It is obvious that the ultrasonic wave itself, affecting the defect in the metal, alters the overall conduction around this defect. However, as thermal waves are involved in conduction alteration, there should have been ultrasonic "responses" associated with such conversion, which did not occur.

In formerly known theories it is stated that surface conduction is directly connected with surface temperature through free electron path time.

It is known that conduction in metal heated above Debye temperature $\left(\theta>0{ }^{\circ} \mathrm{C}\right)$ is directly proportional to free electron path time $(\tau)$ (Drude-Lorentzmodel http://digitus.itk.ppke.hu, 2012):

$$
\sigma=n e^{2} \tau / m
$$

where - $n$ conduction electron number (close to metal atoms, practically independent of temperature); $e$-electron charge $\left(1.6 \times 10^{-9} \mathrm{~K}\right) ; m$ - electron mass $\left(9 \times 10^{-31} \mathrm{~kg}\right.$.), $\tau$-free electron path time $\left(10^{-10}-10^{-13} \mathrm{sec}\right.$. $)$ inversely proportional to Kelvin temperature. 
In time physicists suggested applying phonons as virtual particles (Zeimann, 1962; Kosevich, 1972; Reissland, 1975 ) to describe the functional dependence of conduction to temperature without the parameter $\tau$. The increasing number of thermal phonons is the major factor in describing precisely the conduction behavior dependence to temperature. Nowadays, it is commonly supposed that conduction alteration is possible only under the concentration alteration of virtual particles - thermal phonons; but then arises the question - how does surface temperature vary with ultrasonic frequency? Moreover, this alteration occurs practically simultaneously all over this metal surface!

The heating-cooling performance speed of metal surface has been investigated, but its intensity significantly exceeds that of the intensity applied in our experiments. The paper (Sharkov, Klokov, \& Galkina, 2001) describes the results of the short-pursed laser $\left(\tau_{p}=10 \mathrm{~ns}\right)$ impact (energy $=10^{-9}-10^{-7} \mathrm{~J}$ ) on a gold film of 1500 A.U. thickness. It was established that the cooling time of this film was not more than 150-200 ns, while maximum temperature $60-80^{\circ} \mathrm{K}$ to ambient temperature $\left(3-4^{\circ} \mathrm{K}\right)$ ratio increases up to 20 times $(13 \mathrm{~dB})$. These authors indicated the fact that this laser output power did not fully go into heating the gold film-some beams were reflected spatially, while others were converted into "photonic phonons". Based on the obtained calculations, the effective output power of heating film was not more than $10 \%$. Even such power in calculating per beam area is not less than $200 \mathrm{~W} / \mathrm{mm}^{2}$. In view of the fact that relatively excessive heating temperature is 20 times, it is presupposed that this power could be decreased to $10 \mathrm{~W} / \mathrm{mm}^{2}$ to double the heating when desired conduction alteration is also doubled. But in this case, the ultrasonic wave energy, converting metal defects into further thermal phonons, is 100 times less $\left(5 \mathrm{~W} / \mathrm{cm}^{2}=5 \times 10^{-2} \mathrm{~W} / \mathrm{mm}^{2}\right)$. Nevertheless, experimental results show that speed increase and temperature decrease in the thin metal layer (i.e. "skin layer") could include decile microseconds. It follows that ultrasonic vibration frequency, generating metal defects, could be MegaHertzs. However, there is one debatable question - simultaneous surface conduction alteration all over the surface. In the experiments with laser beams, total temperature area alteration is not more than several $\mathrm{mm}^{2}$, as in our experiments the total sample area included $45 \times 450 \mathrm{~mm}\left(202.5 \mathrm{~cm}^{2}\right)$. To maintain this thermal energy area with excessive heating of several degrees is impossible even that case when the laser involves several Joules. Therefore, proposed thermal surface modulation model is invalid.

Some authors described the above-mentioned possible surface conduction alteration based on other models (Vasilyev \& Lyuboshitz, 1994). For example, the electron Fermi gas model was considered in describing the level of potential energy and chemical potential of electron conductivity inside an undeformed/deformed crystalline metal (solid). It was proved that not only electric field proportional to irrotational deformation gradient, but also volume charge balanced by opposite sign to surface charge occur during inhomogeneous deformation of crystalline metal, which is stated in the following formula:

$$
\mathbf{E}=-\boldsymbol{f} \boldsymbol{K}_{\boldsymbol{e}}\left(z|\boldsymbol{e}| n_{0} \boldsymbol{K}\right)^{-1}
$$

where E-electron energy level inside deformed metal (V); $\boldsymbol{f}$-external force, deforming metal (uniform compression) as volume unit $\left(\mathrm{N} / \mathrm{m}^{3}\right) ; \boldsymbol{K}_{e}$-electron conduction cubic elasticity modulus $\left(1.33 \times 10^{-11}\left(\mathrm{~Pa}^{-1}\right)\right)$; $z$-number of positive ions; $n_{0}$-number of conduction electrons; $\boldsymbol{K}$-deformed body cubic elasticity modulus ( 2 $\left.5 \times 10^{-11}(1 / \mathrm{Pa})\right)$.

Calculated value of electrical field energy depends on the ratio values of cubic electron gas elasticity and deformed metal. This equation is not expressed due to the smallness of results and the fact that this proposed approach is incomplete and cannot substitute detailed analysis, which in its turn, is based on rigorous theory.

There are some contradictions indicating the insolvency of these theoretical implications. Alteration speed of cubic deformation could be directly associated with microwave vibrations, i.e. its source or promotion of cubic metal deformation. Then why do we have the following result - electric field generation does not involve acoustic emission signals, which are well-known characteristics of any alterations in density, crystalline lattice structure and near-edge structure of electron gas volume. Existence of an electrified zone within a conductive body contradicts Van de Graaff generator experiments (Van de Graaff-Generator, 2010). In either case, the physical nature of surface charge alteration is still disputable. However, the above-mentioned surface charge is the most practical theoretical explanation to prove that the surface conduction alteration is a result of the emerging of additional charge carriers besides conduction electrons. It is established that charge carrier concentration alters specifically on the surface, i.e. where it is directly connected with the "skin" layer conduction (Vasilyev \& Lyuboshitz, 1994).

Without discussing this in further details, one should mention their ability to alter the surface conduction of an investigated sample. Given specific zone dimension, generating charges $=V$ is a cube with $m$ face (where $m$ number of interatomic spacing). In this case, the number of electrical field generation events and the additional 
conduction source on the surface is obviously proportional to $m$. Thus, the surface involving these new sources could be written as $R$ (where $R$-face of square surface in interatomic spacing units), where the additional conduction particles should enhance the number of existing conduction electrons on the surface. Moreover, surface $6 R^{2}$ is the surface with double values of conduction sources (new and old ones).

Then, the number of new conduction sources on the surface should equal the number of new charge sources (with opposite sign) in the active zone:

$$
m^{3}=6 R^{2}, R=0,402 m^{3 / 2}
$$

Consequently, observed phenomenon (rate of conduction surface alteration) should depend on the dimensions of the active zones to the power of $3 / 2$. In the case, when the dimensions of the specific active zones close to $1 \mathrm{~mm}$ ( $m=10^{6}$ atomic layers, volume equals $1 \mathrm{~mm}^{3}, R=0.4 \mathrm{E} 9$ atomic layers; $\mathrm{R}=400 \mathrm{~mm}$ ), the volume of the body itself at double conduction alteration should include $0.064 \mathrm{~m}^{3}\left(64 \mathrm{E} 6 \mathrm{~mm}^{3}, 64\right.$ liter).

The proposed calculation does not completely correspond to the physical processes within the metal; the cubic volume excludes the conversion coefficient of cubic ultrasonic energy in the volume alteration of specific electron-gas zone. However, the calculation error is not the subject matter in this case.

This model completely embraces the discovered effect of electron gas interaction with low-frequency ultrasonics, resulting in the effect of MW interaction with metal surfaces, within or on the surface, where there are active defects. Active defects are those zones in a metal which generate additional sources of conduction, and are simultaneously related to specific acoustic sources as their origin time is always connected with the origin of acoustic emission (AE) signals. Though precise correlation of AE signal origin time to variable "skin" layer conduction has not been established, all experimental and research results in determining the generation nature of AE signals (Khonikomb, 1972) are particularly similar to those of our research results.

Moreover, the existing distinction between new and well-known AE methods is possible only in non-contact detection sources of high mechanic strain. In their works Vasilyev and Lyuboshitz indicated the fact that defect control is possible not only on the surface of a metal, but also within the body itself, where earlier invisible defects could have been detected only by contact methods. The most significant conclusion based on the theoretical works of Kurchatovski Institute scientists (Vasilyev \& Lyuboshitz, 1994) is the possible fact that surface charge propagation speed is equal to the speed of light within electron gas medium and less in a vacuum, but significantly surpasses the speed of sound; which is why the observation of surface metal property alterations with a defect (including elastic strain gradient) is practically possible from any metal surface point, as in the above-mentioned experiment. And this fact shows the difference between this method and AE one. In this case, it is essential to further not only theoretical, but also practical investigation, i.e. the design of a sophisticated device for non-contact control of metal unit load level in the motion mode and defect and deterioration rate. An example is the prototype model designed by Gorbunov and Sutorikhin.

\section{Experiment Results}

To investigate the possible defect (cross crack in mid-minor face of an aluminum sample size of $20 \mathrm{x} 40$, length $180 \mathrm{~mm}$ ), the above-described microwave (MW) nondestructive testing device (defectoscope) with domestic CK4-45 spectrum analyzer system SK4-45 was applied.

Piezoelectric transmitters with 44 and $73 \mathrm{KHz}$ excitation were attached to the sample end face. Transmitting excitation was generated from two separate generators. Given transmitting excitation of $44 \mathrm{KHz}$ amplitude was $82-85 \mathrm{~V}$, while at excitation of $73 \mathrm{KHz}-112 \mathrm{~V}$. Special lithol-based lubricant was used for both ultrasonic transmitters to provide close contact with the sample. Instead of metal clamps, special manufactured textolite attachments were applied in the fixation of transmitters to investigate the sample.

The direction of ultrasonic waves was directed to excite a defect (artificial cross crack), i.e. the sound wave angle was parallel to the crack plane. The distance from the aperture horn area to the investigated sample was $120 \mathrm{~mm}$ $\pm 0.3 \mathrm{~mm}$.

To receive maximum signal microwave (MW) parts were adjusted to variation range on the spectrum analyzer screen. At maximum gain and maximum sensitivity of analyzer and spectral bandwidth of $3 \mathrm{KHz}$, the scan speed is $0.3 \mathrm{sec} / \mathrm{screen}$, detector accumulation time-1 sec., linear vertical scale and scan band - 170 and $200 \mathrm{KHz}$. Measured value of sideband components $44,73 \mathrm{KHz}$ was at noise level, but not surpassing this level. However, total component $117 \mathrm{KHz}$ surpassed the noise level up to 2-3 decibel. This can be explained by the fact that the SK4-48 spectrum analyzer is imperfect as it has low response in the operating range base $(0-100 \mathrm{KHz})$, while operating noise coefficient is more than 16 decibel. 
Experiments were conducted not only on the above-described designed prototype device, but also on tension testing devices in the Institute of Physics of Strength and Material Engineering, Siberian Branch of Russian Academy of Science (SB RAS). Samples were silicon-sheet steel of 130x20x0.3mm. The spectrum analyzer was $\mathrm{R} \& \mathrm{CH}$ device with $10 \mathrm{KHz}-4 \mathrm{GHz}$ range. Generator for additional ultrasonic signal was a piezoelectric transducer with $44 \mathrm{KHz}$ frequency and excitation amplitude of $60-70 \mathrm{~V}$. At a stress close to plastic deformation, origin of displayed signal at MW sensor outlet of which the amplitude surpassed the noise up to 5-6 decibel was observed. Small desired signal value can be the result of small reflecting surface size $(20 \mathrm{~mm})$ in comparison to wave length $(8 \mathrm{~mm})$ and small sample thickness $(0.3 \mathrm{~mm})$, i.e. small dimensions of active zones. Research was also devoted to testing active defects in railway wheels in the static mode according to "Start-10" program. Obtained results extended the possible potential performance of the new method. It was established that time parameters of displayed signal at MW sensor outlet significantly depend on active zone positioning, including metal volume. However, in comparison to AE method where single acoustic impulses are detected, the displayed signals are observed in non-continuous development time-interval, i.e. from several seconds to several days. The so-called "blinking" parameter of a displayed signal was detected on the massive railway wheel samples, but, in this case, non-continuous development time-interval was less than 5-8 seconds.

Defect reactivation methods have been developed, which in its turn, has furthered the application of the above-mentioned new method. Reactivation of defects involves significant mechanical and thermal gradients $(400-500 \%$ sec.). This new method exhibits defect activity at minor gradients $(100-150 \% / \mathrm{sec}$.). Besides, activity time parameters of reactivated defects are rather similar to previous ones.

\section{Conclusion}

The newly revealed phenomenon or even discovery of electron gas interaction with low-frequency ultrasonic waves in metals and alloys have been experimentally proven. This method package has a wide application in modern technology. However, lack of fundamental theoretical background of revealed phenomenon is not complication in the practical application and design of an advanced remote indicator of active defects (RIAD) in metals and alloys.

In comparison to well-known non-destructive testing devices the described device includes several positive advantages:

- $\quad$ experimental testing mode involves natural operating conditions without presetting (pump shaft assemblies, turbines, generators, railway wheels, buffer springs, welding seams);

- $\quad$ during loading the object is subjected to additional periodical loading, the frequency range of which is significantly lower than the displayed signal frequency, dissociating from the majority of industrial noise, and registering only one spectral component, as the parameters of additional load ( frequency spectrum and amplitude dependency) are displayed signals;

- $\quad$ MW sensor registers displayed signal at rather significant operating distance alteration. i.e. from the sensor to the investigated sample (1/10 of MW length- $0.5-0.3 \mathrm{~mm})$; there is no other sophisticated variant to differentiate in-going wave and reflecting wave excluding stationary wave effect;

- $\quad$ obtained research results indicate the fact that the development time-interval of active zones is more significant (from several seconds to hundreds of hours) than the time-interval of active acoustic signals; however, this fact makes it possible to detect dangerous stress within the metal after applying critical mechanical loading;

- propagation speed of displayed signals from the dangerous stress zones is close to the speed of light, and tens of thousands higher than ultrasonic speed.

The undeniable practical application of this new device - remote indicator of active defects in metals and alloys embraces such a possibility as detecting not only displayed signals in the motion mode of any object, but also real noise signals. What is more important is the fact that this device detects only those defects which are active ones and are likely to alter the surface conduction (invisible defects as cracks, delamination and incompact adhesion).

Less dangerous defects as pockets, thinning and dents being not active defects cannot be detected by this new device.

Specifically, the present updated device has several variations which can be implemented in the following cases:

1). testing defect technique of railway wheels (radial cracks, cracks around rolling circles, cracks in axles, springs and cranking webs and wheels) in motion mode; this method package includes two attaching parts: one to the train cars - special manufactured device of several ultrasonic sensor transmitters, and the other to the rails - 
several MW sensors;

2). testing technique for determining reliability parameters of wire cables and sucker rod pumps;

3). replacement technique of several high-accuracy (precision) sensors of motion, speed and moment to one RIAD device, as well as, instrumentation for NC machines (Brichkov S. A., \& Sutorikhin V. A., 2008); this can be explained by the fact that metal "fatigue rate" by cutting tools is ultimately the result of displayed signals from MW sensor outlet;

4). method package in investigating metal railway bridges (taking in account such a factor as the extended distance of 1-2 km);

5). diagnostic technique for turbine blades (steam and combustion turbines) to detect early active defects and for operating troubleshooting (rotation speed, torque moment), leading to breakdowns;

6). control device for thermal-insulated pipelines (i.e. basalt coverings) in thermal electric power stations.

This is only the possible application range of the method package relevant to R\&D results and pinpoints the further improvement of the device in the following aspects - sensitivity enhancement and distance extension to determine the reliability of such objects as railway bridges and runways, crude oil \& gas tanks, pipelines and steam pipelines of nuclear and thermal electric power stations.

\section{Acknowledgements}

We would like to thank the specialists and executives of Tomsk Institute of Physics of Strength and Material Engineering, Siberian Branch of Russian Academy of Science (SB RAS) and Research Institute of Non-Destructive Testing, National Research Tomsk Polytechnic University (NR TPU) for their time and efforts in providing a stimulating working environment and their help and encouragement in our research.

\section{References}

Bredow, M., Rumyantsev, V. V., \& Toptigin, I. N. (1985). Classical electrodynamics. Nayka: Moscow., p.400.

Brichkov, S. A., \& Sutorikhin, V. A. (2008). Useful model patent No. 85390. The appliance for precision positioning during the surface treatment of metal products. Priority of a useful model.

Drude-Lorentz model. $\quad$ Retrieved from http://digitus.itk.ppke.hu/ acsurgay/BION_PHYS_ESSZEK_REF/Drude_Sommerfeld/OpticalProp_of_Met als.pdf /

Dudova, N. R., Kaibishev, R. O., \& Valitov, V. A. (2008). Origin of Portevin-Le Chatelier effect in X20H80 alloy. Physics of Metals and Metal Technology, 105(1), 105-112.

Golitsin, B. B. (1960). Selecta (Vol. 1, pp. 95). Academy of Science, USSR: Moscow.

Gorbunov, V. I., \& Sutorikhin, V. A. (1999). Possible control of elastic deformation limits by the microwave (MW) method. Defectoscopy, 7, 75-80.

Gorbunov, V. I., \& Sutorikhin, V. A. (2010). Possibility of non-destructive testing using microwaves. Electronic Journal Technical Acoustics. Retrieved from http://www.ejta.org,16, 9-18.

Gorbunov, V. I., \& Sutorikhin, V. A. (2012). Microwave Nondestructive Testing Method. Applied Physics Research, 4(1), 206-210. http://dx.doi.org/10.5539/apr.v4n1p206

Gorbunov, V. I., Sutorikhin, V. A., \& Ustinov, B. F. (2008). Method patent No. 2372615. The method of registration of acoustic emission signals in metals. Priority of Invention. July 22.

Ju, Y., Saka, M., \& Luo, D. (2002). NDE of Closed Fatigue Crack on the Metal Surface by Microwaves. Department of Mechanical Engineering, Tohoku University, Aoba 01, Aramaki, Aoba-ku, Sendai 980-8579, Japan H. Аbй.

Khonikomb, R. (1972). Plastic deformation of metals. Moscow: Publishing House Mir.

Kosevich, A. M. (1972). Fundamentals of Crystalline lattice mechanics: Moscow.

Leontovich, M. A. (1948). Approximate boundary conditions for electromagnetic fields on surfaces of well-conductive bodies. Research in radio-wave propagation, 2.

Permittivity ( prmtivd) (electricity). (2012). The dielectric constant multiplied by the permittivity of empty space, where the permittivity of empty space $(\varepsilon)$. Retrieved from www.answers.com/topic/permi

Reissland, J. (1975). Physics of phonons. Moscow. 
Sharkov, A. I., Klokov, A. Yu., \& Galkina, T. I. (2001). Generation of excess phonons in semi-conductors and non-conductors of impulse heating metal film: model \& experiment. Physics of Solid Bodies, 43(3), 446-451.

Shibkov, A. A., \& Zolotov, A. V. (2011). Acoustic and optical control of Chernov-Luders bands in aluminummagnesium alloy. Crystallography, 56(1), 147-154.

Ultrasonic. (1970). I. P. M., Goljamin (Ed.), Soviet Encyclopedia (p. 400).

Van de Graaff -Generator. (2010). Electrostatistic generators. Retrieved from nuph.spbstu.ru/minobrminatom/2/accs/.../3.6.html

Vasilyev, B. V., \& Lyuboshitz, V. L. (1994). The Virial theorem and some properties of the electron gas in metals (RAS Session of February 23, 1994). Uspekhi Fizicheskikh Nauk, 164, 367-374.

Vondran, D. (1999). Vector Corrected Noise Figure Measurements. Microwave Journal, 22-38

Zaev, N. E., Avramenko, S. V., \& Lisin, V. N. (2012). Measurement of conductivity current, exciting polarization current. Retrieved from http://www.rusphysics.ru/dissertation/269

Zeimann, J. (1962). Electrons and phonons. Moscow. 